\title{
Adaptive spatial resolution: application to surface plasmon waveguide modes
}

\author{
PETER DEB ACKERE*, PETER BIENSTMAN, ROEL BAETS \\ Photonics Research Group, Department of Information Technology, Gent University, \\ Sint-Pietersnieuwstraat 41, Gent 9000, Belgium \\ (*author for correspondence: E-mail: Peter.Debackere@intec.Ugent.be)
}

Received 11 July 2006; accepted 28 September 2006

\begin{abstract}
In order to simulate surface plasmon waveguide structures we have utilized and improved the adaptive spatial resolution technique and combined it with PML boundary conditions. Using this new technique we have developed a novel concept for an integrated surface plasmon biosensor.
\end{abstract}

Key words: Fourier modal method, adaptive spatial resolution, surface plasmon waveguides, biosensors

\section{Summary}

The Fourier Modal Method (FMM) formulated by Moharam and Gaylord (1983) is known to be slowly converging, especially for TM polarization of metallic lamellar gratings. The correct factorization rules for discontinuous functions derived by $\mathrm{Li}(1996,2002)$ improved the convergence rate for TM polarization (Granet and Guizal 1996; Lalanne and Morris 1996). For thin metallic gratings however, the convergence of the method, even when applying the correct factorization rules, was still problematic. Reformulating the eigenproblem in a new basis can improve convergence rates, as has been shown by Granet (1999), who first introduced the concept of adaptive spatial resolution (ASR). The method consists of a parametric representation of the coordinate axis, which allows a spatially adaptive resolution, increasing the resolution in the neighborhood of the discontinuities of the permittivity function. The original technique was later extended to multilevel profiles (Vallius and Honkanen 2001). Since the FMM can also be applied to calculate waveguide problems (Lalanne and Silberstein 2000), we have modified the parametric reformulation so the formalism could be used to provide reliable estimates for a two-stage method in a eigenmode solver (CAMFR (Bienstman 2004)). In the first stage of the method a coarse estimate of the propagation constant is calculated using the FMM. In a second stage these estimates are refined using a mode-matching method. This technique also allows us to determine the accuracy of the FMM by comparing the coarse estimates of the propagation constants to the actual 
values. Perfectly Matched Layer (PML) boundary conditions (Bienstman and Baets 2002) were also integrated into the formalism.

Using this calculation scheme we have developed a novel concept for an integrated surface plasmon biosensor. The use of surface plasmon resonance (SPR) for biological and chemical sensing is well established. The high sensitivity of this technique to surface phenomena makes it ideal for use in real-time and label-free biosensors where very small changes in refractive index must be detected. In the past decade, several integrated optical SPR sensors have been demonstrated (Harris and Wilkinson 1995; Homola et al. 1997; Čtyrocký et al. 1999), in which thin gold films serving as a platform for the attachment of sensing films are deposited on top of an integrated optical waveguide system. However, all integrated SPR sensors that have been investigated so far are fabricated in a material system with a low refractive index contrast, keeping typical dimensions of waveguides and optical components too large for miniaturization and consequent labon-chip applications. Working with a high refractive index material system such as silicon-on-insulator is a more straight-forward approach to meet the requirements for high-level integration and high-throughput fabrication.

\section{Theory}

The FMM is based on a series representation of the electromagnetic field in the region that contains the grating. This series representation must then satisfy the appropriate boundary conditions (Moharam and Gaylord 1983; Granet and Guizal 1996). Consider the case of a lamellar grating configuration as shown in Fig. 1. A piece-wise homogenuous system with thickness $h$, characterized by a refractive index $n_{2}(x)$, periodic in the $x$ direction with period $d$ and independent of the $y$-coordinate separates the vacuum $(n=1)$ and a homogenuous isotropic medium with refractive index $n_{3}$. This structure is illuminated by a p-polarized monochromatic plane wave with a

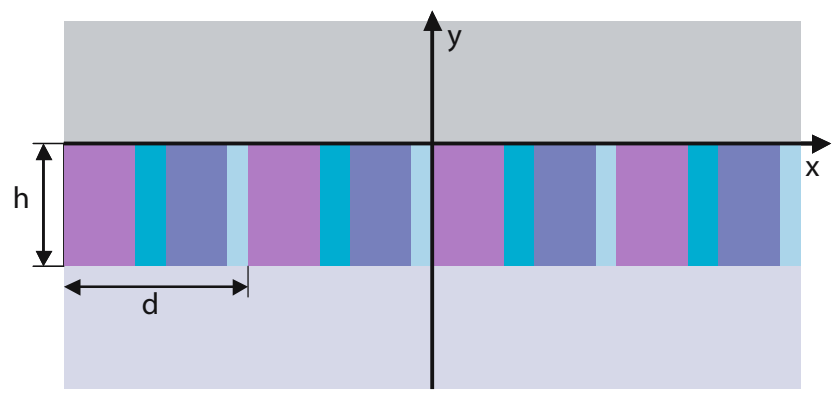

Fig. 1. Setup for the FMM. 
vacuum wavelength $\lambda$, angular frequency $\omega$ and wave number $k$ at normal incidence. The unknown function is the $y$-component of the magnetic field $H_{y}(x, z)$. In each region, which we refer to by the subscript $i, i \in\{1,2,3\}$, the field can be represented by the following superposition of eigenmodes:

$$
H_{i y}(x, z)=\sum_{q}\left[A_{i q}^{+} \mathrm{e}^{-i k r_{i q} z}+A_{i q}^{-} \mathrm{e}^{i k r_{i q} z}\right] \phi_{i q}(x)
$$

where $A_{i q}^{+}$and $A_{i q}^{-}$are modal-field amplitudes and $r_{i q}$ and $\phi_{i q}(x)$ are modal eigenvalues and modal eigenfunctions, which are determined by the boundary-value problem

$$
\begin{aligned}
L_{i}(x) \phi_{i q}(x) & =k^{2} r_{i q}^{2} \phi_{i q}(x), \\
\phi_{i q}(x+d) & =\phi_{i q}(x),
\end{aligned}
$$

where $L_{i}(x)$ is a second order differential operator derived from Maxwell's equations and only depending on the $x$ variable.

$$
L_{i}(x)=n_{i}^{2}(x)\left(\frac{\partial}{\partial x}\left(\frac{1}{n_{i}^{2}(x)} \frac{\partial}{\partial x}\right)+k^{2}\right) .
$$

In the vacuum and the substrate, the $H_{i y}$ functions are given by Rayleigh expansions. The only remaining problem is to compute the eigenmodes $\phi_{2 q}(x)$ and the eigenvalues $r_{2 q}^{2}$ in the grating region. For that purpose we first expand the eigenmodes in terms of plane waves

$$
\phi_{2 q}(x)=\sum_{m} \phi_{2 m q} \mathrm{e}^{-i k a_{m} x},
$$

and then project Equation (2) onto the Fourier basis $\mathrm{e}^{-i 2 \pi m(x / d)}$. We thus obtain a matrix for which the eigenvalues and eigenvectors are being sought. The above described theory is known as the classical FMM.

In order to solve the convergence problems, we first need to take a look as to what is causing them. The main reason for slow convergence is that spatial resolution remains uniform within a grating period whatever the permittivity may be (Granet 1999; Vallius and Honkanen 2001). The parametric FMM solves this problem by choosing a coordinate system in such a way that the mapping of space fits the variation of the periodic function of interest. In order to do so we need a function $x=x(u)$ so that spatial resolution is increased at the discontinuity points. Around these points a given variation $\Delta u$ of $u$ should result in a smaller variation $\Delta x$ of $x$. In other words, the coordinate transformation function maps non-uniformly spaced points along the $x$ axis in the physical domain to uniformly spaced points in the transferred $u$ domain. We present here the coordinate $x$ as a 
function of $u$ and the transition points are denoted by $x_{l}$ in the $x$ space and by $u_{l}$ in the $u$ space. Between the transitions $x_{l-1}$ and $x_{l}$ we use the function $x_{l}(u)$ for the mapping between different domains. In comparison with the original formulation (Granet 1999) the formalism was modified so that the resolution near the discontinuities is increased even more (due to the fact that $a_{3}$ depends only on the difference between $x_{l}$ and $x_{l-1}$ and not on the total thickness $d$ ), and the discontinuities in the new basis can differ from the discontinuities in the old basis. In comparison with the work of Vallius and Honkanen (2001) the period $d^{\prime}$ in the new basis can differ from the original period $d$, which allows us to stretch the entire structure.

$$
x_{l}(u)=a_{1}+a_{2} u-\frac{n a_{3}}{2 \pi} \sin \left(\frac{2 \pi\left(u-u_{l-1}\right)}{u_{l}-u_{l-1}}\right)
$$

with

$$
\begin{aligned}
a_{1} & =\frac{u_{l} x_{l-1}-u_{l-1} x_{l}}{u_{l}-u_{l-1}}, \\
a_{2} & =\frac{x_{l}-x_{l-1}}{u_{l}-u_{l-1}} \\
a_{3} & =x_{l}-x_{l-1} .
\end{aligned}
$$

The above relation defines a cartesian coordinate system $(u, y, z)$ with a scale factor along the $x$-axis. We then introduce a new vector basis $\mathbf{e}_{u}=$ $(\mathrm{d} x / \mathrm{d} u) \mathbf{e}_{x}$, the other two unit vectors $\mathbf{e}_{y}$ and $\mathbf{e}_{z}$ being unchanged. The newly defined function of $u$ is periodic with period $d^{\prime}$ which can be different than the period of the permittivity function and its derivative is minimal at the discontinuities. It can then be shown that the $L_{i}$ operator becomes

$$
L_{i}(u)=\frac{1}{b_{i}(u)}\left(\frac{\mathrm{d} x}{\mathrm{~d} u}+\frac{\partial}{\partial u}\left(\frac{1}{a_{i}(u)} \frac{\partial}{\partial u}\right)\right)
$$

with

$$
b_{i}(u)=\frac{h(u)}{n_{i}^{2}(u)}, \quad a_{i}(u)=h(u) n_{i}^{2}(u), \quad h(u)=\frac{\mathrm{d} x}{\mathrm{~d} u} .
$$

We can obtain a matrix relation by following the same way as we did in the classical FMM. The matrix for which the eigenvalues are being sought is given by

$$
\left[\mathbf{L}_{i}\right]=\left[\mathbf{b}_{i}\right]^{-1}\left[[\mathbf{h}]-[a]\left[\mathbf{a}_{i}\right]^{-1}[a]\right]
$$


where $[\mathbf{h}]$ is the matrix formed by the Fourier coefficients of $h$ such that

$$
h_{m n}=h_{m-n}=\frac{1}{2 d} \int_{-d^{\prime}}^{d^{\prime}} h(u) \mathrm{e}^{-i 2 \pi(m-n) \frac{u}{d^{\prime}}} \mathrm{d} u .
$$

Similar definitions hold for $\left[\mathbf{a}_{i}\right]$ and $\left[\mathbf{b}_{i}\right],[a]$ is the diagonal matrix formed by $a_{q}$, defined by

$$
a_{q}=q \frac{\lambda}{d^{\prime}}
$$

The main advantage of this change of coordinate is that we no longer need the Fourier coefficients of the exact refractive index profile, but of the transformed refractive index profile $h(u) \epsilon_{r}(u)$. The choice of coordinate mapping thus has a direct consequence on the convergence of the Fourier series. By comparing the convergence of the Fourier series we arrived at three fairly obvious choices. The first possibility is to divide the period $d$ in $l$ equal sections, we shall refer to this method as the uniform solver. The second possibility is to chose the transitions so that the peak height of the function $h(u) \epsilon_{r}(u)$ is equal in each section, this will be referred to as the adaptive solver. The third possibility introduces a new period $d^{\prime}$ and divides this period into equal sections, this shall be referred to as the extended uniform solver. As an illustration we depicted the transformed dielectric profile $h(u) \epsilon_{r}$ for a thin metallic layer $\left(40 \mathrm{~nm}, \epsilon_{r}=-19-0.5301 j\right)$, surrounded by a dielectric cladding layer $\left(1 \mu \mathrm{m}, \epsilon_{r}=4\right)$ and the Fourier approximation truncated after 10 (red), 25 (orange), 50 (green) and 100 (blue) modes. Only the real part of the dielectric function is shown in these figures. One can clearly see that the uniform solver and the extended uniform solver show the most promising convergence.

\section{Numerical example}

We have investigated the convergence properties of the new method and compared them to the original formulation of the FMM and Granet's formulation (1999).

The setup consists of a thin metallic layer (width $40 \mathrm{~nm}, n=0.0608-$ $4.3593 j$ ) surrounded by dielectric cladding layers (width $1 \mu \mathrm{m}, n=2$ ). If the metallic layer is thin enough, the surface plasmon modes guided by the interfaces become coupled due to field tunneling through the metal, thus creating supermodes. In general, only two purely bound TM modes are guided by a such a structure. In the plane perpendicular to the direction of propagation the electric field of these modes is comprised of a single component, normal to the interface, having either a symmetric or 

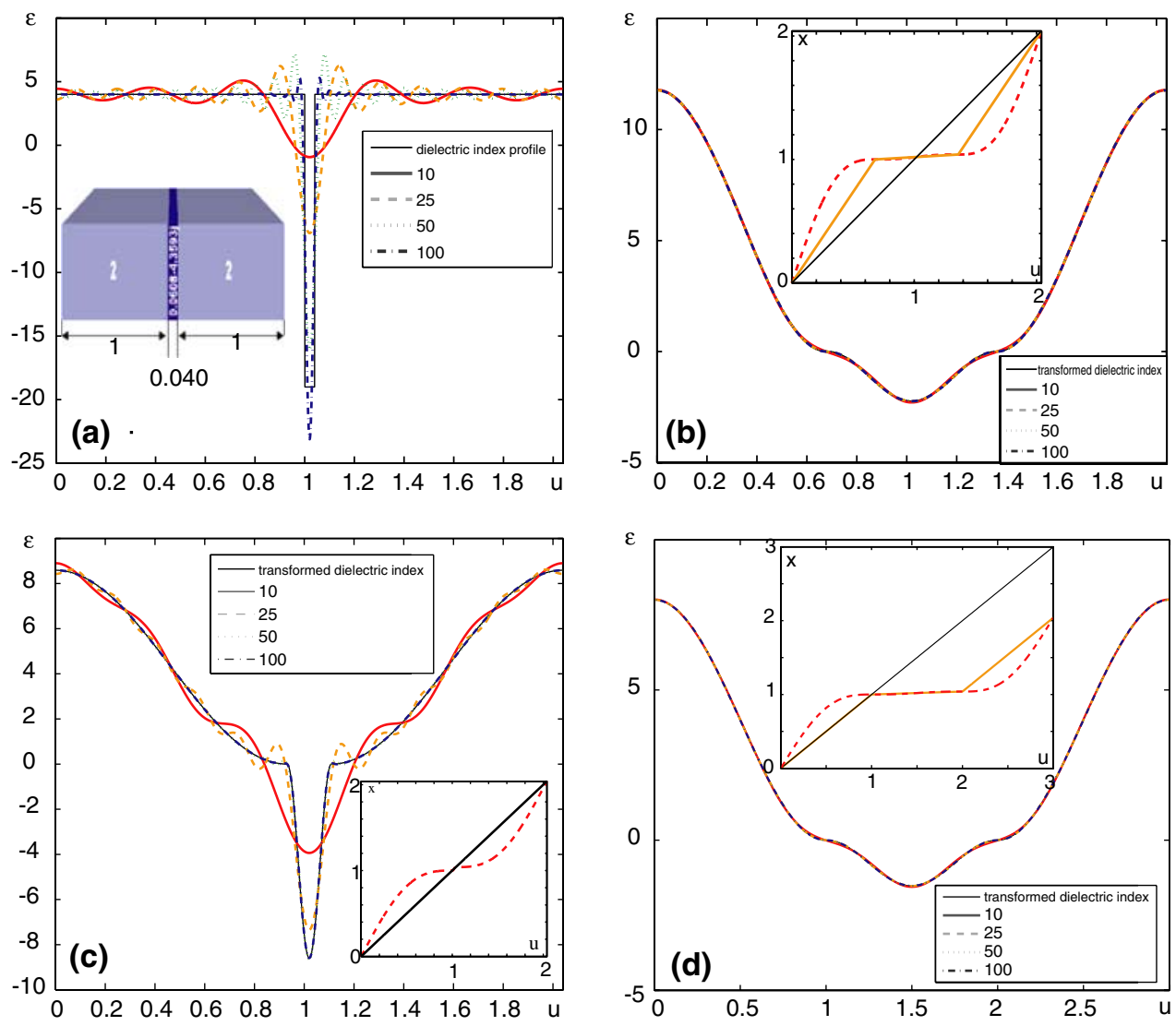

Fig. 2. Comparison of the different coordinate transformation schemes. (a) Profile of the relative dielectric constant of a thin metallic layer, the configuration is shown in the inset. (b) Transformed relative dielectric profile of the uniform solver algorithm, the inset depicts the coordinate transformation for this configuration. (c) Transformed relative dielectric profile of the adaptive solver algorithm, the inset depicts the coordinate transformation for this configuration. (d) Transformed relative dielectric profile of the extended uniform algorithm, the inset depicts the coordinate transformation for this configuration.

assymmetric distribution across the waveguide. While the symmetric mode extends into the cladding layers, the asymmetric mode is tightly bound to the metallic waveguide and will be extremely lossy. In order to test the convergence of both new methods we made use of the symmetries and placed the entire structure between two magnetic walls, thus selecting the asymmetric plasmon polariton mode. In order to reduce reflections from the top magnetic wall we have also incorporated PML in the simulations implemented as a complex cladding thickness. We calculated the eigenvalues by using a growing number of the truncation order and compared the convergence of the real part of the first eigenvalue. The result of this simulation is plotted in Fig. 3. Due to the small feature size the classical FMM converges slowly. Since the old parametric formulation (Granet 1999) does not 


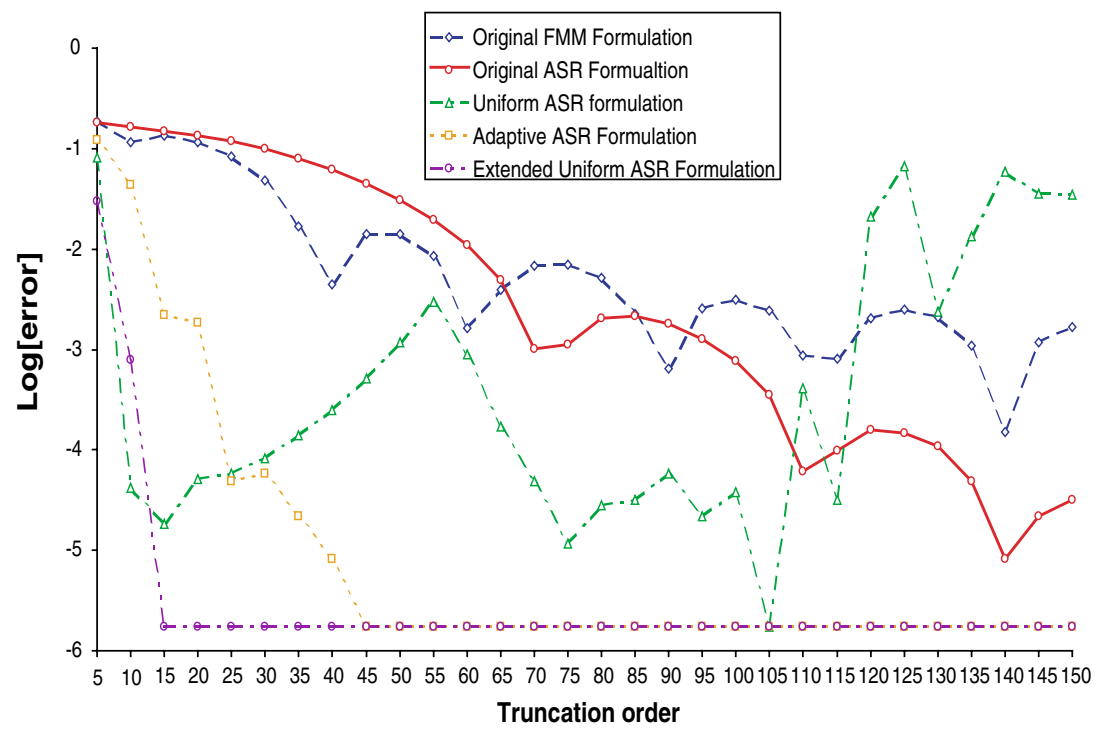

Fig. 3. Convergence of the first order eigenmode of a metallic waveguide with PML boundary conditions.

allow the spreading of the small features in the $u$ space, the Gibb's phenomenon will prevent small detail to be resolved correctly, which has a negative impact on the convergence of this method. Due to the presence of PML boundary conditions the uniform algorithm fails to converge. This is due to the fact that the algorithm reduces the distance between the guiding metallic layer and the boundaries and thus the PML has a detrimental influence on the convergence of the guided modes. By stretching the entire structure the thickness of the cladding layers can be maintained and the convergence is optimal as can be seen in Fig. 3

\section{Application: a surface plasmon biosensor}

Using this calculation scheme we have designed and simulated a novel concept of a surface plasmon biosensor. Only the basic design and principle of the device will be discussed here, for a detailed analysis we refer to Debackere et al. (in press). A scheme of the surface plasmon interferometer is depicted in Fig. 4. The interferometer consists of a gold layer embedded in the silicon membrane on top of a supporting silica layer. When a dielectric TM-polarized mode guided by the silicon membrane slab waveguide enters the structure and reaches the gold, two surface plasmon modes are launched, one at the upper and one at the lower interface of the gold layer. Since the refractive indices of the upper and the lower cladding layers 


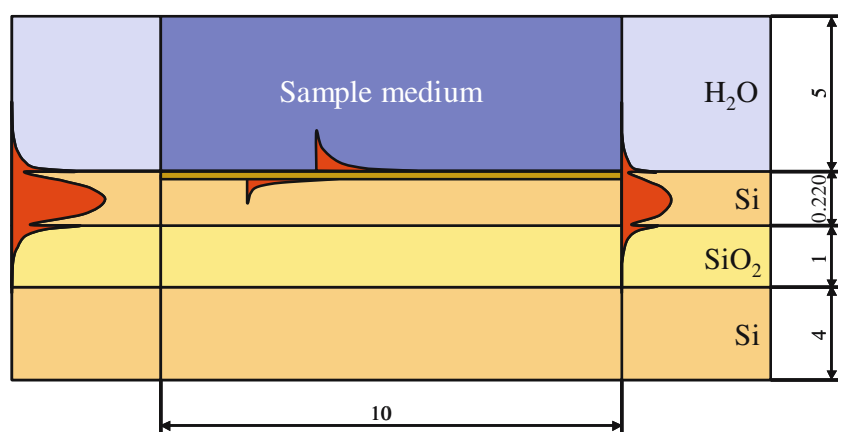

Fig. 4. Schematical setup of the proposed structure, all dimensions in $\mu \mathrm{m}$.

are sufficiently different, these modes cannot couple. Therefore, their phase velocities are entirely determined by the refractive index of the upper and lower dielectric respectively. At the end of this section, interference of the two surface plasmon modes results in a dielectric mode launched in the output waveguide. This explains the sensing functionality of the interferometer: a change in the refractive index of the medium above the gold layer results in a phase difference between the two surface plasmon modes and consequently, in a change of output intensity.

Fig. 5 illustrates the interferometric nature of our device. For a sensing section of length $10 \mu \mathrm{m}$, the transmitted intensity of the fundamental TM mode of the silicon slab waveguide is plotted as a function of refractive index of the sample medium. For this simulation, we have chosen a wavelength of $1.55 \mu \mathrm{m}$, which is in the near-infrared region and suitable for biosensing applications. When the upper and lower surface plasmon modes arrive in phase at the end of the sensing section, constructive interference leads to maximal transmission. However, for certain values of the sample refractive index, the phase difference between the two modes equals $\pi$, resulting into destructive interference and a minimum in the transmission curve.

Because the device is based on interference, the resonance of the system can be tuned to a multitude of required wavelength ranges, or refractive index ranges. The main parameters governing the response of this device are the length of the sensing region, the thickness of the $\mathrm{Si}$ waveguide in the sensing region, and the thickness of the Au layer.

By varying these three parameters we have optimized the sensor depicted in Fig. 4 so that the transmission would be minimal for a refractive index of the sample medium of 1.33 , and a wavelength of $1.55 \mu \mathrm{m}$. In the optimized design the thickness of the silicon membrane is equal to $101 \mathrm{~nm}$, and the length of the device is equal to $6.055 \mu \mathrm{m}$. Simulation results for this device are shown in Fig. 6. 


\section{ASR: APPLICATION TO SURFACE PLASMON WAVEGUIDE MODES}

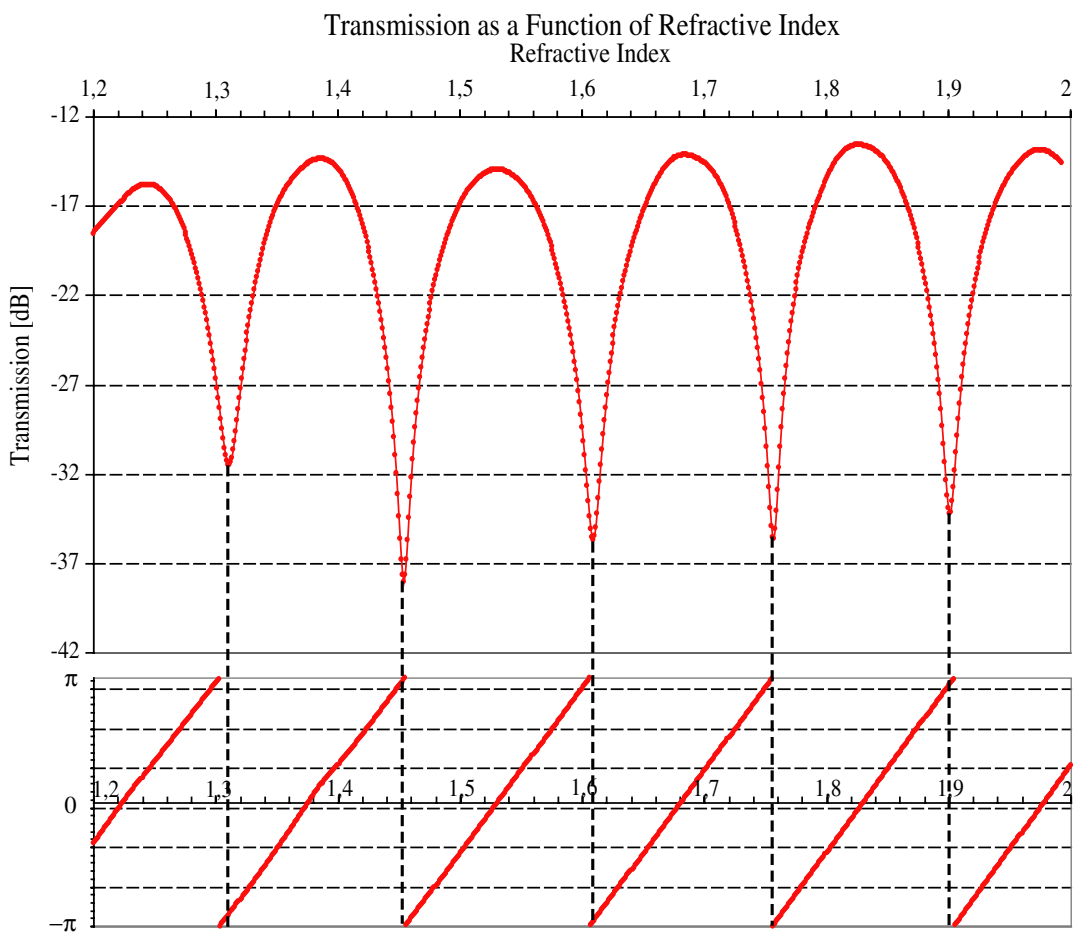

Fig. 5. Transmission of the structure depicted in Fig. 4 as a function of refractive index. The length of the structure is $10 \mu \mathrm{m}$.

Transmission as a function of Refractive index

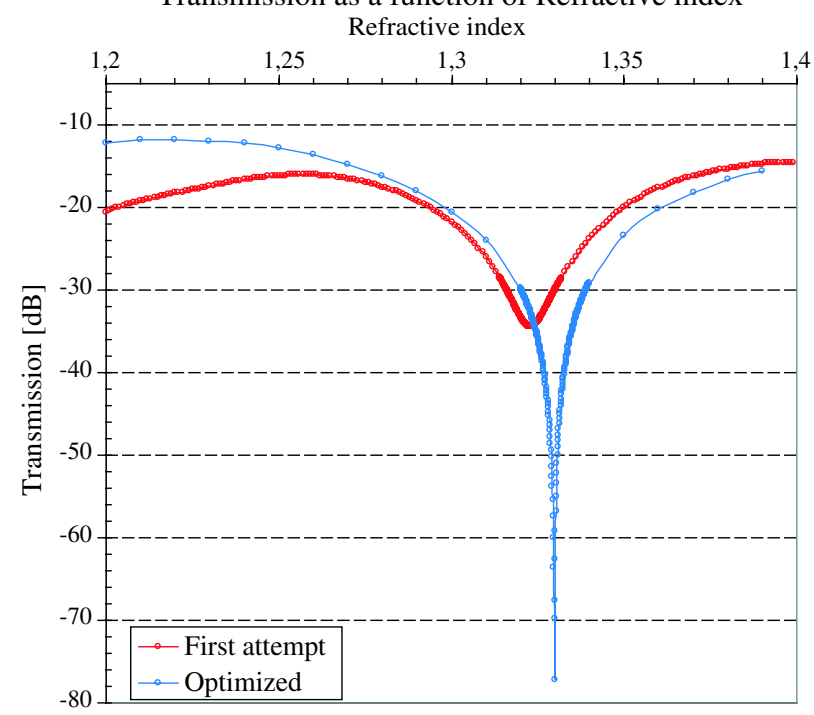

Fig. 6. Simulation results for the optimized structure. 
If we work at a fixed wavelength and vary the refractive index of the sample medium, we can calculate that sensor sensitivity for this device reaches values of $10,000 \mathrm{~dB} / \mathrm{RIU}$ (refractive index unit). In conjunction with an optoelectronic system which can measure changes in the optical power of $0.01 \mathrm{~dB}$, variations in the refractive index as small as $10^{-6}$ can be measured. This value is comparable with that of other integrated surface plasmon sensors (Čtyrocký et al. 1999), the dimensions however are two orders of magnitude smaller. This means that the smallest amount of a certain molecule that can be detected will also be two orders of magnitude smaller than current integrated surface plasmon sensors.

\section{Conclusion}

In order to solve the convergence problems associated with very thin metal layers, we modified the methods derived by Vallius and Honkanen (2001). Three different approaches towards improving the convergence of this method have been described, and of these only one, namely the extended uniform method, has been withheld, as it works well with PMLs based on a complex cladding thickness. This methods should allow one to simulate plasmon polariton modes with eigenmode solvers instead of FDTD methods, thus drastically reducing the simulation time and the reliability of these simulations.

With the use of this method we have developed a novel concept for an integrated surface plasmon biosensor. The device has a number of interesting benefits. First, the device is two orders of magnitude smaller than conventional surface plasmon waveguide sensors, due to the integration into a high-index contrast material system. Second the device is highly tunable, making an excellent candidate for a vast number of applications.

The authors believe this novel concept to be an important step toward a fully integrated surface plasmon lab-on-chip solution.

\section{Acknowledgments}

This work was carried out in the context of the GOA project (Ghent University), and was supported by the Belgian IAP PHOTON network. P. Bienstman acknowledges the Flemish Fund for Scientific Research (FWO-Vlaanderen) for a postdoctoral fellowship. 


\section{ASR: APPLICATION TO SURFACE PLASMON WAVEGUIDE MODES}

\section{References}

Bienstman, P. Opt. Quant. Electron. 36(1/3) 5, 2004.

Bienstman, P. and R. Baets. Opt. Quant. Electron. 34(5/6) 523, 2002.

Čtyrocký, J., J. Homola, P.V. Lambeck, S. Musa, H.J.W.M. Hoekstra, R.D. Harris, J.S. Wilkinson, B. Usievich and N.M. Lyndin. Sensor Actuator B $\mathbf{5 4}$ 66, 1999.

Debackere, P., S. Scheerlinck, P. Bienstman and R. Baets. Opt. Exp. 14(16) 7063, 2006.

Granet, G. JOSA 16(10) 2510, 1999.

Granet, G. and B. Guizal. JOSA 13(5) 1019, 1996.

Harris, R.D. and J.S. Wilkinson. Sensors Actuators B 29 261, 1995.

Homola, J., J. Čtyrocký, M. Skalský, J. Hradilová and P. Kolářová. Sensors Actuators B 38-39 286, 1997.

Li, L. JOSA 13(9) 1870, 1996.

Li, L. JOSA Commun. 19(7) 1443, 2002.

Lalanne P. and M. Morris. JOSA 13(4) 779, 1996.

Lalanne P. and E. Silberstein. Opt. Lett. 25(15) 1092, 2000.

Moharam, M.G. and T.K. Gaylord. JOSA 73 451, 1983.

Vallius T. and M. Honkanen. Opt. Exp. 10(1) 24, 2001. 


\section{SpringerLink}

Athens Authentication Point

\section{Recognized as:}

Universiteit Gent

(378-89-845)

\section{Welcome!}

To use the personalized features of this site, please log in or register.

If you have forgotten your username or password, we can help.

My SpringerLink

Marked Items

Alerts

Order History

Saved Items

All

Favorites
Content Trpes Subjects

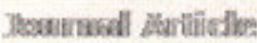

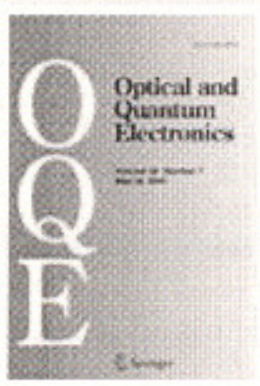

Adaptive spatial resolution: application to surface plasmon waveguide modes

Journal

Optical and Quantum

Publisher Springer Netherlands

ISSN 0306-8919

(Print) 1572$817 \times$ (Online)

Subject Engineering

Status OMUINEFIRST

DOI $\quad 10.1007 / \mathrm{s} 11082$

006-9010-3

Online Date Tuesday,

December 19, 2006
Add to marked items Add to shopping cart Add to saved items Recommend this article
Peter Debackere ${ }^{1}$, Peter Bienstman ${ }^{1}$ and Roel Baets ${ }^{1}$

(1) Photonics Research Group, Department of Information Technology, Gent University, Sint-Pietersnieuwstraat 41, Gent, 9000, Belgium

Received: 11 July 2006 Accepted: 28 September 2006 Published online: 19 December 2006

Abstract In order to simulate surface plasmon waveguide structures we have utilized and improved the adaptive spatial resolution technique and combined it with PML boundary conditions. Using this new technique we have developed a novel concept for an integrated surface plasmon biosensor.

Keywords Fourier modal method - adaptive spatial resolution - surface plasmon waveguides - biosensors

Peter Debackere

Email: Peter.Debackere@intec.Ugent.be References secured to subscribers.
Find more options

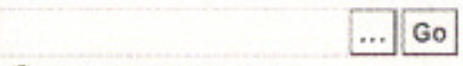

Within this journal

(-) Within all content

Export this articie

Export this article as RIS|Text

Text

PDF

The size of this document is 712 kilobytes. Although it may be a lengthier download, this is the most authoritative online format.

Open: Entire document 